\title{
Looking ahead, looking behind
}

\author{
James P. Evans, MD, PhD
}

T his issue of Genetics in Medicine is devoted to celebrating the 20th anniversary of the founding of the American College of Medical Genetics and as such features articles that look back over the past 20 years and ones that try to look ahead. The history of the College's founding is summarized by David Rimoin (page 179), one of the midwives as the American College of Medical Genetics emerged from the virtual womb back in 1991. We have also managed to dig up some pictures of the founders but hasten to add that the editorial board of Genetics in Medicine is not responsible for any ravages of time that might be evident.

When we envisioned an anniversary issue of Genetics in Medicine, we felt that although looking back over the College's history was appropriate, it would be most exciting to focus primarily on the future. A harder task, to be sure, but given the fact that our field is often discussed in terms of its future impact, it seems worth considering what the next 20 years may have in store.

The advent of technology capable of affordable whole genome sequencing (WGS) is sure to dominate our field for some time to come. But precisely how WGS will play out in the clinical arena is an open question at this time. Thus, we feature two commentaries which in some ways represent the two poles of a vision for clinical application of next generation sequencing. I personally do not agree with either commentary, but the job of an editor is not to simply publish material one agrees with. Ideally, it is to provoke thought and frame issues; goals which I think that these two commentaries accomplish well.

In the first, Richard Sharp, an eminent and perceptive thinker regarding the ethical, legal, and social implications of genetics, argues for "downsizing" our efforts due to formidable consent challenges and the tremendous data-handling problems that are sure to arise when one begins to grapple with terabytes of information (page 191). Although these issues are certainly thorny and will require innovation and wisdom to successfully confront, I personally do not think that they present insurmountable barriers to the rather near-term implementation of WGS in the clinic. The use of arrays and panels of genes, a scaled-down approach for which Sharp argues, is limited by our profound myopia: what genes should be in a given panel and what alleles represented in any given array? Rather, I suspect the way forward is through the application of WGS to defined patient populations in a learn-as-you-go context. And while that may sound cavalier, it is precisely how medicine has always progressed and is a legitimate approach - as long as we keep a focus on studying what we are doing and on generating evidence to guide our course. Any analysis of the kinds of information derived in any individual's WGS illustrates our profound ignorance of what the majority of it means. And within that ignorance lies the way forward. Because the majority of information

From the Departments of Genetics and Medicine, University of North Carolina, Chapel Hill, North Carolina.

James P. Evans, MD, PhD, Departments of Genetics and Medicine, University of North Carolina, Chapel Hill, NC. E-mail: jpevans@med.unc.edu.

Disclosure: The author declares no conflict of interest.

DOI: 10.1097/GIM.0b013e318210b0a7 derived from a WGS will be utterly clinically insignificant (as we have no idea of its clinical meaning), the majority of it cannot - and should not-be in a patient's primary medical record. Only what is known to be clinically meaningful (an extreme minority) should be confirmed and placed there. Many institutions are now constructing data warehouses to act as informational buffers to house large data files that do not belong in a patient's primary record, such as the petabytes of imaging information that are now routinely generated. A similar arrangement is ideally suited to storing genomic information and could allow the gradual movement of clinically relevant findings to the primary record as evidence dictates.

Similarly, although formidable, consent issues do not seem overwhelming to me. Yes, the sheer volume of information that patients will need to grapple with is large. But patients and doctors have been grappling with large amounts of ambiguous, unexpected and potentially sensitive information for many years. I think it is time to jettison the last vestiges of genetic exceptionalism and realize that the field of medicine, as a whole, is fraught with sensitive findings, unpleasant surprises, and privacy concerns discovered in the course of routine workups. Genetics is no different. By carving up the genome into manageable pieces, we can deal with this problem in partnership with our patients. I suspect that patients are up to the job of understanding that some unexpected WGS results must be conveyed back to them because there are actionable medical consequences. Indeed, we perform head magnetic resonance imaging scans every day and some of those reveal unexpected tumors. However, we do not consent patients before a magnetic resonance imaging simply because we might find out medically important but surprising things. Patients need to be informed at the outset of WGS that unexpected findings may be uncovered and that information of a sensitive nature (e.g., APOE status) will inevitably be generated. Such a discussion can be rendered manageable by addressing it in terms of the broad categories of information that will be found. For the categories containing those things (like $A P O E$ ) that have no current medical actionability, patients can be offered educational materials to enable them to decide whether they want to learn of their results within that category or would rather let it slide.

Sharp may well be right; perhaps we should downsize our activities. But if we do, I am afraid we will never learn how to deal with such information. Sooner or later, we have to cross the divide and begin to deal clinically with large amounts of genetic information. My suspicion is that by downsizing to panels of genes and arrays, we get the worst of both worlds; i.e., we limit the useful information that we will be deriving but will still be faced with qualitatively complex problems of unexpected and ambiguous information. Moreover, I suspect that the train has left the station and that efforts to downsize our ambition are realistically doomed. Finally, I am optimistic about the promise of WGS, especially in the realm of diagnostics.

However, I am not nearly as optimistic as Drmanac in his commentary (page 188), I agree that sequencing costs will continue to plummet and that the cost of sequencing an individual's whole genome will be far less than $\$ 1000$ relatively soon. But I see little support for the conjecture that this will 
herald "significant improvements in human health and reduced health care costs." The reality is that most human diseases are simply not primarily genetic. Thus, although genetic investigation is sure to tell us important things about the underlying mechanisms of disease, it is unlikely to revolutionize the treatment of most human maladies in the near- to mid-term. The most salient value of WGS will likely be in a significantly enhanced ability to diagnose those (relatively rare) diseases that result from discrete, highly penetrant genetic lesions. Although a better understanding of the human organism in health and disease is sure to be forthcoming from the current "genomic revolution," the history of medicine teaches us that practical benefit from improved scientific understanding is hard-won and is an agonizingly slow process. Finally, there are few examples of reduced cost from almost any serious technological breakthrough in medicine; reduced costs are usually balanced by broader and deeper implementation. I doubt that genomics will prove the exception.

The excessive optimism found in Drmanac's commentary, I am afraid, will run up against the hard and cruel shoals of evidence-as it should. If there is one overarching lesson to be learned from the past 20 years of medicine in general, it is that we must be guided not by wishful thinking and theoretically attractive ideas, but rather by actual evidence of benefit. Genomics must be "pulled" into medicine by evidence that it actually helps our patients, not "pushed" into medicine because it seems like it ought to work or is just so cool we can hardly contain our enthusiasm. Indeed, we must contain that enthusiasm or we will repeat the same mistakes made by our medical brethren when they prescribed hormone replacement therapy for postmenopausal women en masse, administered lidocaine drips to peri-infarct patients and subjected countless American men to an ill-conceived mass experiment in PSA screening. As we move forward to implement astounding technologies and basic discoveries for patient benefit, we must seek to generate evidence to test our ideas every step of the way. This is not an easy task. We cannot expect randomized controlled trials to address every issue; resources and time do not permit it and the rarity of many genetic findings will preclude it. Rather, we must develop ongoing analytic frameworks by which evidence can be parsed to the best of our ability by admittedly imperfect means and use constant revision, review, and questioning of our trajectory to help us do a better and better job of sorting the wheat from the chaff.

Finally, as I look ahead to the next 20 years, I am not really worried about a shortage of medical geneticists. As someone at a recent meeting I attended said "I'll believe there's a shortage of medical geneticists when I hear it from someone other than a medical geneticist." Ouch! But indeed, such a real shortage would be the best thing that could ever happen to our field, as it would finally "pull" us into the general world of medicine. Heaven knows our "pushing" has had little impact. And if such a real shortage ever materializes, the incentives will finally be in place to pay for training programs, ensure adequate reimbursement, and attract new recruits. As it stands, bringing the benefits of genetic advances to our patients will be a team effort and I suspect we are up to the job along with our compatriots, the genetic counselors. Our proper current goal is simple: we must work to demonstrate ways in which we and our shiny new tools can actually help provide better health for the people of the world.

But who really knows what is in store for our field over the next 20 years? As we muse on that topic, it would be well to heed what Niels Bohr said: "Prediction is very difficult, especially about the future." The real fun will lie in being part of that future, whether through developing new technologies, glimpsing the significance of noncoding DNA, or guiding patients through the bewildering maze that is modern medicine. Invoking the words of Niels Bohr seems appropriate in that I have often thought that the privilege of being a geneticist in these times of excitement for our field must echo the fevered awe felt by physicists at the turn of the 20th century when new technologies and a new understanding of the world made for constant intellectual ferment.

What we can be certain of is that our field will continue to surprise us throughout the next two decades. Although I am sure that we will not be up to anticipating those surprises (or they would not be so-named), I can assure you that Genetics in Medicine will endeavor to inform the College (and a hopefully broader and broader readership) of the latest advances and to provide a forum by which those immersed in Medical Genetics can grapple with a field sure to continue moving at a breakneck pace. 